\title{
Erbium luminescence imaging of infrared surface plasmon polaritons
}

\author{
E. Verhagen, ${ }^{\text {a) }}$ A. L. Tchebotareva, ${ }^{\text {b) }}$ and A. Polman \\ Center for Nanophotonics, FOM Institute for Atomic and Molecular Physics, Kruislaan 407, \\ 1098 SJ Amsterdam, The Netherlands
}

(Received 22 December 2005; accepted 7 February 2006; published online 24 March 2006)

\begin{abstract}
We demonstrate a new technique to spatially map the propagation and damping of infrared surface plasmon polaritons (SPPs) on metal films using optically active erbium ions as a probe of the SPP field. The bound SPP mode propagating along the Ag/glass interface of a $96 \mathrm{~nm}$ thick Ag film on glass is excited by illuminating a subwavelength hole array in the metal with $1.49 \mu \mathrm{m}$ light. By imaging the $1.53 \mu \mathrm{m}$ photoluminescence of Er ions positioned in the glass at a distance of $60 \mathrm{~nm}$ from the Ag/glass interface in a confocal microscope, a SPP beam was observed to propagate along a broad stripe waveguide, with a characteristic propagation length of $76 \mu \mathrm{m}$. This technique provides a useful tool to study the characteristics of SPP modes at metal-dielectric interfaces in a wide range of geometries. (C) 2006 American Institute of Physics. [DOI: 10.1063/1.2189188]
\end{abstract}

A possible route toward the miniaturization and integration of optical components on a planar substrate involves the employment of surface plasmon polaritons (SPPs) on metallic structures. SPPs are evanescent electromagnetic waves propagating along a metal/dielectric interface, coupled to coherent oscillations of free electrons in the metal. ${ }^{1}$ Their large wave vectors and confinement to the metal interface make these modes promising candidates for the guiding of light at a very small length scale. However, because of intrinsic damping in the metal, SPPs exhibit significant loss. In recent years much effort has been undertaken to visualize the propagation of SPPs along structured metal films and metal stripe waveguides with thicknesses of a few tens of nanometers deposited on glass substrates, which show characteristic propagation lengths of tens to hundreds of micrometers for visible and infrared wavelengths. ${ }^{2,3}$ Such geometries can sustain two types of SPP modes: those that have the largest field components at the metal/air surface, and those that are concentrated at the metal/glass interface. The former lose energy to leakage radiation into the glass substrate, because their wave vector is smaller than that of light in glass, whereas the latter are bound in all media. Most imaging techniques, such as photon scanning tunneling microscopy (PSTM) ${ }^{4,5}$ and leakage radiation microscopy, ${ }^{6,7}$ have been aimed at the detection of the (leaky) modes propagating along the metal/air interface.

Here we present a novel method to image purely bound SPP modes in the infrared wavelength regime, by using erbium ions as a probe of the SPP field intensity at the metal/ glass interface. $\mathrm{Er}^{3+}$ ions implanted in glass show considerable absorption around $1.48 \mu \mathrm{m}$ due to intra- $4 f$ transitions. The evanescent field of SPPs propagating along the Ag/glass interface of an Ag film on glass can excite Er ions placed at close proximity to this interface. Their photoluminescence (PL) emission around $1.53 \mu \mathrm{m}$ can be detected in the far field. The use of fluorescent probes to image SPPs was first reported by Ditlbacher et al. ${ }^{8,9}$ In contrast to the fluorescent dye molecules employed in that work, erbium ions do not experience bleaching and thus allow for a more quantitative

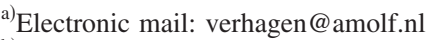

${ }^{b}$ Present address: Huygens Laboratory, Leiden University, P.O. Box 9504, 2300 RA Leiden, The Netherlands.
}

analysis, and the glass substrate itself can serve as a host material.

Because SPPs propagating along the metal/glass interface of an Ag stripe waveguide on glass cannot be excited in the conventional Kretschmann configuration, we use coupling by periodic surface modulations to achieve the needed momentum matching. To excite this mode locally by illumination from the air side of the Ag film, the incident beam is focused onto a subwavelength hole array fabricated in the metal. The enhanced transmission through such structures is believed to be related to the generation of SPPs on both interfaces of the metal film, ${ }^{10}$ and the coupling condition for a square array of holes with principal axes $x$ and $y$ can be given by

$$
\mathbf{k}_{\mathrm{SPP}}=\mathbf{k}_{\|}+\frac{2 \pi}{a}(i \hat{x}+j \hat{y}),
$$

where $\mathbf{k}_{\text {SPP }}$ is the SPP wave vector, $\mathbf{k}_{\|}$is the component of the incident wave vector along the metal surface, $a$ is the pitch of the hole array, and $i$ and $j$ can take positive or negative integer values. Even though the SPP dispersion on the array differs dramatically from that on a smooth film, finite-sized hole arrays have been demonstrated to launch SPPs propagating on a smooth metal film away from the structured area. $^{11,12}$

A fused silica substrate (suprasil, Heraeus) was doped with $\mathrm{Er}$ by $150 \mathrm{keV} \mathrm{Er}{ }^{+}$ion implantation with a fluence of $7 \times 10^{15} \mathrm{Er} / \mathrm{cm}^{2}$, resulting in a peak Er concentration of 3 at. \%. The estimated average depth of the Er ions in the glass was $60 \mathrm{~nm}$, and the standard deviation of the depth distribution was $13 \mathrm{~nm}$, as calculated with the Monte Carlo program TRIM. ${ }^{13}$ This distance to the Ag film allows efficient SPP-Er coupling without much PL quenching by the metal. ${ }^{14}$ The sample was then annealed in a vacuum for $1 \mathrm{~h}$ at $900{ }^{\circ} \mathrm{C}$ to remove any implantation damage. ${ }^{15}$ Electronbeam lithography and lift-off were used to pattern square arrays of holes and stripe waveguides in an Ag film. The $96 \mathrm{~nm}$ thick Ag film was deposited onto the substrate by electron-beam evaporation after the deposition of a $4 \mathrm{~nm}$ thick Si adhesive layer to facilitate the lift-off process without introducing an extra absorptive layer at the metal/glass interface. 


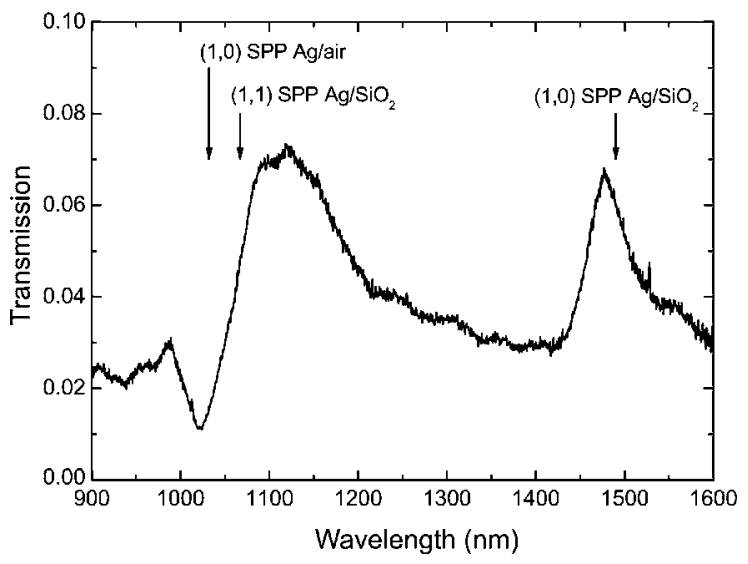

FIG. 1. Normal-incidence optical transmission spectrum of a square array of holes with a $1.02 \mu \mathrm{m}$ pitch in a $96 \mathrm{~nm}$ thick Ag film on fused silica glass. The arrows indicate the wavelengths that would satisfy the coupling condition (1) for various $(i, j)$, assuming SPP wave vectors equal to those on smooth films.

Figure 1 shows the normal-incidence transmission spectrum of a square array of holes with a pitch of $1.02 \mu \mathrm{m}$. The hole shape was roughly square, with a size of $\sim 0.3 \mu \mathrm{m}$. Clear peaks are observed that can be associated with the resonant excitation of SPPs on each of the interfaces. The arrows mark the wavelengths for which the coupling condition (1) is satisfied on the interfaces of the metal film for different sets of $(i, j)$, using the SPP dispersion relation expected for a smooth film. The transmission peak around $1.48 \mu \mathrm{m}$ is attributed to the $( \pm 1,0)$ excitation of SPPs at the metal/glass interface.

A schematic of the setup used is displayed in Fig. 2(a). Excitation light with either $s$ or $p$ polarization at a wavelength of $1.49 \mu \mathrm{m}$ from a $45 \mathrm{~mW}$ InGaAsP laser diode was coupled into a single-mode polarization-maintaining fiber, and focused on the sample surface under a controllable angle to the surface normal with a fiber focuser [numerical aperture $(\mathrm{NA})=0.1]$, resulting in a $10 \mu \mathrm{m}$ diam spot. Light was collected through the substrate with a $20 \times$ objective (NA $=0.4$ ) and passed through a polarizer and a bandpass filter

(a)

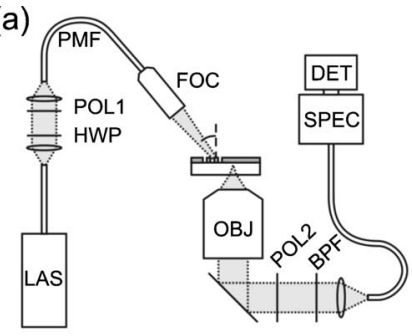

(c)

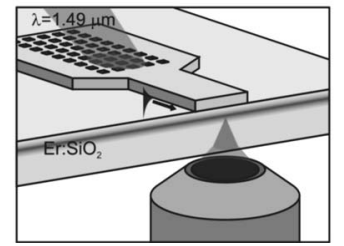

(b)

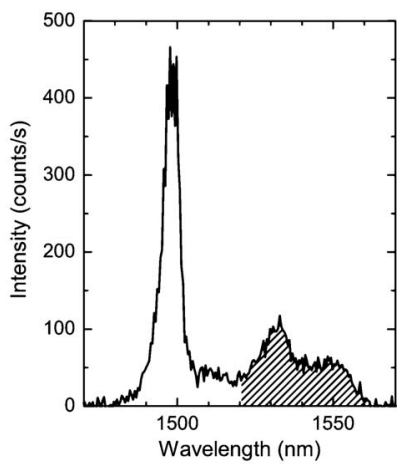

FIG. 2. (a) Schematic overview of the setup used. LAS: $1.49 \mu \mathrm{m}$ laser diode; HWP and POL1: half wave plate and polarizer to control excitation polarization; PMF: polarization maintaining fiber; FOC: fiber focuser (NA $=0.1)$; OBJ: $20 \times$ objective (NA=0.4); POL2 and BPF: polarizer and bandpass filter to reduce scattered laser intensity; SPEC: spectrograph; DET: InGaAs photodiode array detector. (b) A typical spectrum collected with a $3 \mathrm{~s}$ acquisition time, showing scattered $1.49 \mu \mathrm{m}$ laser light and Er PL (shaded). (c) Schematic of the principle of the imaging configuration.

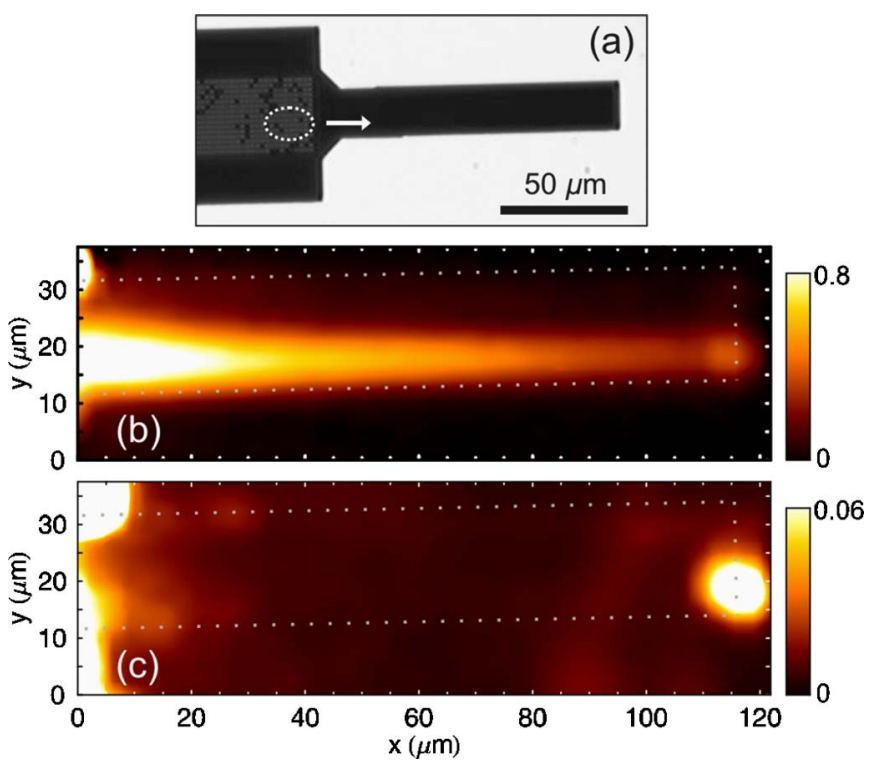

FIG. 3. (Color online) (a) Bright-field transmission microscopy image of the $\mathrm{Ag}$ microstructure that was studied. Indicated are the position of the excitation spot on the hole array (dashed ellipsoid) and the direction of SPP propagation. (b) and (c) Two-dimensional confocal microscopy images for p-polarized excitation of (b) the Er PL intensity between 1520 and $1570 \mathrm{~nm}$, and (c) the intensity around the laser wavelength between 1480 and $1510 \mathrm{~nm}$. The plane of incidence is parallel to the $x$ axis, and the excitation spot is located to the left of the images. The color scale of (b) indicated at the right-hand side is normalized to 0.8 times the maximum intensity in the figure, and the color scale of (c) is normalized to 0.06 times the maximum intensity in the figure.

transmitting wavelengths between 1.508 and $1.556 \mu \mathrm{m}$, to reduce the collection of scattered laser light. Spectra were recorded by dispersing the collected Er luminescence with a spectrograph onto a liquid-nitrogen cooled InGaAs photodiode array detector (Princeton Instruments OMA V). A wavelength-independent background of 500 counts/pixel due to the dark current of the detector was measured and subtracted from the spectra. Although the incident angle was large enough for the directly transmitted laser beam not to be collected by the objective, a significant amount of $1.49 \mu \mathrm{m}$ light was detected due to scattering at the $\mathrm{Ag}$ microstructures. A typical spectrum showing peaks attributed to this laser scattering and Er emission around $1.53 \mu \mathrm{m}$ is displayed in Fig. 2(b). The sample stage was scanned together with the excitation beam with respect to the collection objective in a confocal microscope to obtain a two-dimensional spectrally resolved map of the Er emission and pump scattering. Figure 2(c) shows an illustration of the imaging technique and sample geometry.

Considering the position of the peak in the transmission spectrum (Fig. 1) corresponding to $(1,0)$ excitation of SPPs at the $\mathrm{Ag} / \mathrm{glass}$ interface for normal incidence light, it is expected that on a similar hole array with a pitch of $2 \mu \mathrm{m}$, this mode would be excited with $1.49 \mu \mathrm{m}$ light at an incident angle of $45^{\circ}$ with respect to the surface normal. Laser light was focused under such conditions on a hole array with a pitch of $2 \mu \mathrm{m}$ and a hole size of $\sim 0.75 \mu \mathrm{m}$ that was connected to a $115 \mu \mathrm{m}$ long, $20 \mu \mathrm{m}$ wide $\mathrm{Ag}$ stripe waveguide directed along the plane of incidence. A bright-field optical transmission microscopy image of the sample is shown in Fig. 3(a), with the position of the excitation spot indicated by the dashed circle. The images obtained by integrating the spectra collected on the Ag stripe in the spectral region of the o AIP license or copyright, see http://apl.aip.org/apl/copyright.jsp 


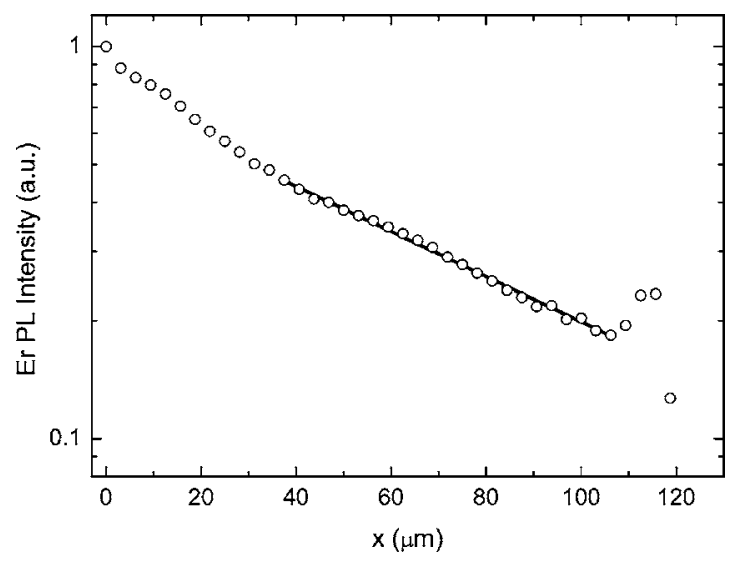

FIG. 4. Er PL intensity integrated over cross sections of the beam observed in Fig. 3(b) as a function of distance $x$ along the SPP waveguide (open circles), displayed on a logarithmic scale. The solid line through the data for $x<37 \mu \mathrm{m}$ is an exponential fit with a characteristic decay length of $76 \pm 1 \mu \mathrm{m}$.

Er emission $(1.52-1.57 \mu \mathrm{m})$ and around the laser wavelength $(1.48-1.51 \mu \mathrm{m})$ are shown in Figs. 3(b) and 3(c), respectively, for $p$-polarized excitation. The outline of the rectangular stripe region, which starts at $x=0 \mu \mathrm{m}$, is indicated by the dashed lines. A beam is clearly seen in the $\mathrm{Er}$ signal [Fig. 3(b)], that remains confined to the Ag stripe. It is attributed to the generation of a SPP mode propagating along the Ag/glass interface that excites the implanted Er ions. For $s$-polarized excitation (data not shown), only a very weak beam was observed with an intensity 30 times smaller than that for $p$-polarized excitation, which is consistent with the fact that SPPs are only excited using $p$-polarized light. The small intensity that is observed for $s$-polarized excitation is attributed to a small instrumental error in the control of the excitation polarization. It is important to note that a beam as observed for the Er luminescence in Fig. 3(b) is not observed in the image of scattered radiation at the laser wavelength [Fig. 3(c)]. Instead, this image is dominated by scattering at the edges of the structure. Although SPPs are expected to scatter to radiation at the end of the waveguide, ${ }^{4}$ it is not possible to attribute the large intensity at the laser wavelength at $x=115 \mu \mathrm{m}$ solely to SPP scattering, since it was also observed for $s$-polarized excitation, albeit with a somewhat lower intensity. It is probably partly caused by the scattering of laser light at a local sample imperfection.

To estimate the damping of the SPP, it is necessary to take into account the slightly varying width of the SPP beam along the waveguide. Figure 4 shows the decay of the Er PL intensity integrated across the beam, measured along the direction of SPP propagation. It is clear that especially at the start of the waveguide the decay is not monoexponential. This may be due to the fact that Er ions close to the launching spot are also excited by light that is diffracted by the hole array and propagates in the glass at a small angle to the $\mathrm{Ag}$ film. Fitting an exponential decay through the data for $x$ $>37 \mu \mathrm{m}$, displayed as a solid line in Fig. 4, gives a 1/e propagation length of $76 \pm 1 \mu \mathrm{m}$. Observation of the decay along longer distances is required to further confirm the exponential nature of the SPP decay.

The expected propagation length of the SPP mode propagating at the substrate side of a smooth $\mathrm{Ag}$ film on fused silica, with the Si adhesive layer taken into account, can be obtained from a calculation of the power dissipation of a harmonic oscillator dipole in the vicinity of such a film using a model developed by Ford and Weber. ${ }^{16}$ The power dissipated as a function of the in-plane wave vector shows a resonance around the SPP wave vector, and the half-width of this resonance is determined by the SPP damping. Using a wavelength of $1.49 \mu \mathrm{m}$ and the dielectric constant of the Ag film measured with ellipsometry $(\varepsilon=90+3.8 i$ at $\lambda=1.49 \mu \mathrm{m}$ ), the expected propagation length thus obtained is $135 \mu \mathrm{m}$. The fact that the observed propagation length of $76 \mu \mathrm{m}$ is smaller suggests that the scattering of SPPs to film roughness and grain boundaries contributes significantly to the SPP damping.

In conclusion, we have visualized the propagation of a locally excited SPP mode along the metal/glass interface of a $96 \mathrm{~nm}$ thick Ag waveguide. The SPPs were generated by the illumination of a subwavelength hole array with $1.49 \mu \mathrm{m}$ light incident from the air side of the film. The SPP beam was observed to decay with a characteristic decay length of $76 \mu \mathrm{m}$. The technique demonstrated here can be of interest to study the propagation of other SPP modes as well, such as the long-range SPP (LR-SPP) mode that is supported by a metal film embedded in a symmetric dielectric background. This method can be employed to locally probe the SPP field intensity at positions in a wide range of plasmonic nanostructures that are not readily accessible with other imaging techniques.

The authors thank Henkjan Gersen for providing tools for dipole power dissipation calculations. This work was made possible by the fabrication and characterization facilities of the Amsterdam nanoCenter. It is part of the research program of the "Stichting voor Fundamenteel Onderzoek der Materie (FOM)," which is financially supported by the "Nederlandse organisatie voor Wetenschappelijk Onderzoek (NWO)."

${ }^{1}$ H. Raether, Surface Plasmons (Springer, Berlin, 1988).

${ }^{2}$ B. Lamprecht, J. R. Krenn, G. Schider, H. Ditlbacher, M. Salerno, N. Felidj, A. Leitner, F. R. Aussenegg, and J.-C. Weeber, Appl. Phys. Lett. 79, 51 (2001).

${ }^{3}$ C. Marquart, S. I. Bozhevolnyi, and K. Leosson, Opt. Express 13, 3303 (2005).

${ }^{4}$ P. Dawson, F. de Fornel, and J.-P. Goudonnet, Phys. Rev. Lett. 72, 2927 (1994).

${ }^{5}$ J.-C. Weeber, J. R. Krenn, A. Dereux, B. Lamprecht, Y. Lacroute, and J.-P. Goudonnet, Phys. Rev. B 64, 045411 (2001).

${ }^{6}$ B. Hecht, H. Bielefeldt, L. Novotny, Y. Inouye, and D. W. Pohl, Phys. Rev. Lett. 77, 1889 (1996).

${ }^{7}$ A. Hohenau, J. R. Krenn, A. L. Stepanov, A. Drezet, H. Ditlbacher, B. Steinberger, A. Leitner, and F. R. Aussenegg, Opt. Lett. 30, 893 (2005). ${ }^{8}$ H. Ditlbacher, J. R. Krenn, N. Felidj, B. Lamprecht, G. Schider, M. Salerno, A. Leitner, and F. R. Aussenegg, Appl. Phys. Lett. 80, 404 (2002).

${ }^{9}$ H. Ditlbacher, J. R. Krenn, G. Schider, A. Leitner, and F. R. Aussenegg, Appl. Phys. Lett. 81, 1762 (2002).

${ }^{10}$ T. W. Ebbesen, H. J. Lezec, H. F. Ghaemi, T. Thio, and P. A. Wolff, Nature (London) 391, 667 (1998)..

${ }^{11}$ D. S. Kim, S. C. Hohng, V. Malyarchuk, Y. C. Yoon, Y. H. Ahn, K. J. Yee, J. W. Park, J. Kim, Q. H. Park, and C. Lienau, Phys. Rev. Lett. 91, 143901 (2003).

${ }^{12}$ E. Devaux, T. W. Ebbesen, J.-C. Weeber, and A. Dereux, Appl. Phys. Lett. 83, 4936 (2003).

${ }^{13}$ J. F. Ziegler, J. P. Biersack, and U. Littmark, The Stopping and Range of Ions in Solids (Pergamon, New York, 1985).

${ }^{14}$ J. Kalkman, L. Kuipers, A. Polman, and H. Gersen, Appl. Phys. Lett. 86, 041113 (2005).

${ }^{15}$ A. Polman, J. Appl. Phys. 82, 1 (1997).

${ }^{16}$ G. W. Ford and W. H. Weber, Phys. Rep. 113, 195 (1984). 